Nouvelles perspectives en sciences sociales

Revue internationale de systémique complexe et d'études relationnelles

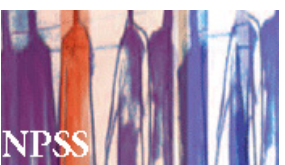

\title{
Un verre à moitié plein ou un verre à moitié vide
}

\section{Brahime Larouz}

Volume 9, numéro 1, novembre 2013

Sur le thème : linguistique et complexité

URI : https://id.erudit.org/iderudit/1024042ar

DOI : https://doi.org/10.7202/1024042ar

Aller au sommaire du numéro

\section{Éditeur(s)}

Prise de parole

\section{ISSN}

1712-8307 (imprimé)

1918-7475 (numérique)

Découvrir la revue

\section{Citer cet article}

Larouz, B. (2013). Un verre à moitié plein ou un verre à moitié vide. Nouvelles perspectives en sciences sociales, 9(1), 199-218.

https://doi.org/10.7202/1024042ar

\section{Résumé de l'article}

Énoncer une phrase, c'est faire référence à un état de choses dont on parle; mais cet état de choses n'est pas, en général, complètement symbolisé par la phrase : pour déterminer ce dont on parle, il faut prendre en considération non seulement la phrase qu'on énonce, mais aussi le contexte d'énonciation; et certains éléments dans la phrase ont justement pour effet d'indiquer quel aspect de la situation d'énonciation doit être considéré pour savoir ce dont on parle. 


\title{
Un verre à moitié plein ou un verre à moitié vide
}

\author{
BraHIME LAROUZ \\ Université Moulay Ismaïl, Maroc
}

et article a pour objet de récuser une thèse psychologisante,
communément admise, selon laquelle dire qu'un verre est à moitié plein, c'est être optimiste; et dire qu'un verre est à moitié vide, c'est être pessimiste. Nous essayerons de démontrer que la langue est fondamentalement argumentative : elle parle d'ellemême pour défendre ou justifier tel ou tel usage discursif. Les éléments extralinguistiques ne permettent pas toujours de donner une explication largement satisfaisante des énoncés du langage; il faudrait plutôt tabler sur ce qui inhérent à la langue ou tout ce qui en ressort en tant que structure ancrée dans telle ou telle situation d'énonciation.

Ce faisant, nous présenterons d'abord quelques aspects de l'argumentation, comme cadre théorique sur lequel nous fonderons notre analyse sémantico-pragmatique. La théorie de l'argumentation conçue par Jean-Claude Anscombre et Oswald Ducrot rend compte des indications pragmatiques ou rhétoriques qu'elle intègre dans la langue en tant que système de communication ${ }^{1}$. Cette idée de pragmatique ou de rhétorique intégrées ${ }^{2}$ est une

\footnotetext{
1 Jean-Claude Anscombre et Oswald Ducrot, L'argumentation dans la langue, Bruxelles, Mardaga, 1983.

2 Dans la mesure où elles sont "intégrées ", cette pragmatique et cette rhéto-
} 
mise en question de l'opposition entre sémantique et pragmatique telle qu'elle a été présentée par la conception positiviste. Pour beaucoup d'auteurs positivistes, l'ordre d'étude est nécessairement linaire : on commence par la syntaxe, on passe à la sémantique pour en venir finalement à la pragmatique ${ }^{3}$.

On aurait donc le schéma ci-dessous illustrant l'idée que chaque composant "ignore " sur quoi le composant précédent a travaillé, et " connaît " uniquement les résultats de son travail :

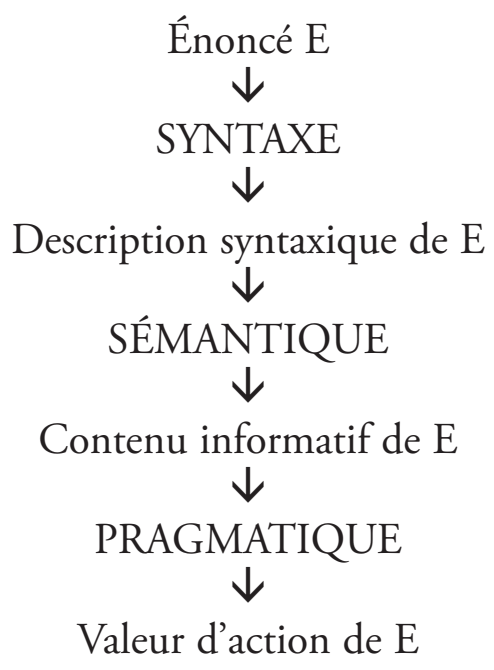

Considérons les deux derniers niveaux (sémantique et pragmatique) les seuls qui nous intéressent : la thèse en litige affirme qu'il n'y a pas dans l'énoncé, ni dans la description syntaxique, de marques essentiellement pragmatiques, marques qu'on ne pourrait donc interpréter que par le troisième composant. Ainsi, si certains mots ou tournures possèdent telle ou telle efficacité pragmatique, ce ne serait possible qu'en vertu des informations qu'ils apportent : un signe n'agit qu'à travers ce dont il informe.

Ducrot conteste cette conception : pour lui, dans la plupart

rique ne relèvent donc pas de ce qui est appelé " composant rhétorique " (voir le chapitre 4, dans Oswald Ducrot, Dire et ne pas dire. Principes de sémantique linguistique, Paris, Herman, 1972), mais de ce qui y apparaît comme "composant linguistique".

3 Charles W. Morris, Foundations of the Theory of Signs, Chicago, University of Chicago Press, 1948. 
des énoncés, il existe des marques qui déterminent la valeur pragmatique de ces énoncés indépendamment de leur contenu informatif, d'où l'idée de pragmatique intégrée 4 .

À ce propos, la pragmatique intégrée, dont il est question ici, ne rendra pas compte de tous les phénomènes qualifiés traditionnellement de pragmatiques, mais seulement de ceux relevant de la conventionalité linguistique, à savoir ceux qui sont inscrits dans la structure même de la langue. Il existe donc des indications ou des instructions (pour reprendre les termes de Ducrot) qui orientent ou indiquent la façon dont on doit interpréter les énoncés.

L'interprétation d'un énoncé doit rendre compte de cet ensemble d'instructions qui permettent d'expliquer la façon d'attribuer un sens aux énoncés. Les opérateurs et les connecteurs argumentatifs sont le lieu de manifestation privilégié de l'instruction argumentative. Un opérateur, par exemple, ne restreint pas seulement les actes d'argumentation réalisables à l'aide d'un énoncé $E^{\prime}$ dans la mesure où ces actes d'argumentation ne subissent plus de telle restriction lorsqu'ils sont réalisables à l'aide d'un énoncé $\mathrm{E}$ qui ne contient pas cet opérateur; de même un connecteur n'indique pas seulement qu'un acte d'argumentation a lieu entre tel argument et telle conclusion. En effet, un opérateur donne également des instructions sur l'orientation argumentative de l'énoncé, en fonction desquelles les possibilités argumentatives de l'énoncé se voient apparemment limitées. Ainsi, dire :

1 - Il est presque 8 heures.

2 - Il n'est que 8 heures.

3 - Il est bien 8 heures.

indique quelle est l'orientation argumentative de l'énoncé de base "il est 8 heures" qui semble neutre argumentativement. "Presque » indique que 1 est orienté vers « le tard » et non vers "le tôt " (l'enchaînement avec " dépêche-toi » étant plus naturel qu'avec « ne te presse pas »). Dans 2 , "ne...que " indique qu'il

4 Jean-Claude Anscombre et Oswald Ducrot, op. cit. 
y a encore du temps et non qu'il manque. Enfin, dans 3, "bien » indique que du temps a passé (un enchaînement avec " tu vas être en retard " étant plus acceptable qu'avec « tu as du temps»).

De même un connecteur argumentatif comme " mais " ou " même » indique quelle est l'orientation des arguments qu'ils articulent :

4 - Gaston est intelligent mais brouillon.

5 - Gaston est intelligent et même travailleur.

En 4, " mais " indique que le premier argument (Gaston est intelligent) est orienté positivement, et que le deuxième argument (Gaston est brouillon) l'est négativement. En 5, "même " indique que le premier argument (Gaston est intelligent) et le deuxième argument (Gaston est travailleur) sont coorientés.

L'anti-orientation (dans le cas de " mais ") ou la coorientation (dans le cas de "même ") détermine donc la nature de l'acte d'argumentation réalisé par la séquence " $\mathrm{p}$ mais $\mathrm{q}$ " ou " $\mathrm{p}$ même q ", c'est-à-dire la nature, d'une part, de l'argument (orienté positivement ou négativement) et, d'autre part, la conclusion (positive ou négative).

Hormis ces instructions argumentatives, d'autres instructions dites énonciatives, parce qu'associées aux marques énonciatives, donnent des indications sur la façon d'intégrer l'énonciation au sens de l'énoncé. En effet, lorsque A énonce devant B :

6 - Je pars puisque vous me le dites. ce que justifie "puisque " n'est pas le départ de A, mais l'annonce de son départ, c'est-à-dire son énonciation (le fait qu'il réalise un acte d'énonciation de valeur illocutoire informative). Plus généralement, "puisque " indique qu'il enchaîne non pas sur le contenu, mais sur l'énonciation de l'énoncé précédent.

Outre ces instructions (argumentatives et énonciatives), il existe un troisième type d'instructions dites " discursives »: il s'agit des indications sur le type d'enchaînement compatible avec les propriétés sémantiques, pragmatiques ou argumentatives de l'énoncé. Considérons, à titre d'exemple, l'instruction selon laquelle l'enchaînement se fait sur le contenu posé, et non sur le 
contenu présupposé5 ou encore l'instruction selon laquelle l'enchaînement se fait sur la valeur littérale d'une implicitation conversationnelle particulière et non sur sa valeur implicite ${ }^{6}$ comme le montrent respectivement les énoncés 7 et 8 :

7 - X ne fume plus.

- parce qu'il est malade.

- *donc il fumait avant.

7’ - posé : X ne fume pas actuellement.

- présupposé : avant, X fumait.

8 - La soupe manque de sel.

- *pour que je puisse commencer à manger.

- parce que c'est A qui l'a faite.

8' - sens littéral = la soupe manque de sel.

- sens implicite $=$ passe-moi le sel.

De telles instructions peuvent se combiner. Dans ce cas, on pourra prédire que 9 est argumentativement mal formé, car l'enchaînement ne satisfait pas les instructions argumentatives imposées par " mais" :

9 - Achille est intelligent mais paresseux. Il faut donc absolument lui confier ce poste de haute responsabilité.

La pragmatique n'est donc pas surajoutée à la description sémantique : elle lui est plutôt intégrée. Qu'en est-il maintenant pour les indications rhétoriques?

Ducrot part de la constatation suivante : on ne peut pas utiliser n'importe quelle expression en faveur de n'importe quelle conclusion $^{7}$. Que l'on considère le texte suivant :

10 - Peu de Marocains respectent la loi (presque $20 \%$ ).

$5 \quad$ Voir Oswald Ducrot, Dire et ne pas dire..., op. cit.

$6 \quad$ Voir Eddy Roulet, Langue maternelle et langues secondes : vers une pédagogie intégrée, Hatier-Crédif, 1980.

7 Oswald Ducrot, Dire et ne pas dire..., op. cit. 
La parenthèse (presque $20 \%$ ) présentée ici comme un argument autorisant l'appréciation portée dans la principale (peu de Marocains respectent la loi) rend l'énoncé bizarre. Bien plus, cela a une résonnance étrange même si l'on tient pour faible une proportion légèrement inférieure à $20 \%$. Dans ce cas, les expressions attendues à l'intérieur de la parenthèse seraient "pas tout à fait ", " un peu moins de ", " pas plus de ". Pour comprendre le comportement de "presque ", il faut renoncer à faire de la parenthèse un argument, et la considérer au contraire comme une restriction, en y introduisant un " mais " (mais quand même presque $20 \%$ ).

En poussant un peu plus loin l'analyse, $\operatorname{Ducrot}^{8}$ a établi deux classes de modificateurs quantitatifs selon que leur présence dans la parenthèse exige ou n'exige pas de conjonction d'opposition analogue à " mais » :

Exigent «mais»

- presque

- plus de

- un peu plus de

- pas moins de

- guère moins de

- au moins
N'exigent pas « mais», ou même l'interdisent

- pas tout à fait

- pas plus de

- un peu moins de

- moins de

- guère plus de

- au plus

- à peine

- seulement

Il existe donc des expressions dont l'utilisation discursive est soumise à certaines restrictions impossibles à déduire de leur valeur informative. Plus généralement, dès qu'un énoncé les contient, on voit apparaitre des contraintes sur le type de conclusions en faveur desquelles il peut être utilisé.

La langue n'est plus considérée d'une manière indépendante de ses conditions de mise en pratique : en effet, le sens de certaines formes linguistiques dépend de leur utilisation. Énoncer

8 Jean-Claude Anscombre et Oswald Ducrot, op. cit. 
une phrase, c'est faire référence à un état de choses dont on parle, mais cet état de choses n'est pas, en général, complètement symbolisé par la phrase. Pour déterminer ce dont on parle, il faut prendre en considération non seulement la phrase qu'on énonce, mais aussi le contexte d'énonciation; et certains éléments dans la phrase ont justement pour effet d'indiquer quel aspect de la situation d'énonciation doit être considéré pour savoir ce dont on parle. Considérons cet exemple :

11 - Maintenant sortons d'ici!

On ne peut pas déterminer la portée significative de cet énoncé abstraction faite du contexte d'énonciation puisque le mot "sortons " renvoie à la personne qui l'énonce : " maintenant " au moment de l'énonciation et " ici » au lieu de l'énonciation. Ces mots ont donc pour effet de renvoyer à leur utilisation.

Outre le sens de ces formes linguistiques, qui renvoient à l'énonciation, on doit reconnaître que cette dernière elle-même est porteuse de sens : à côté de ce qui est dit, il y a ce qui est signifié par le fait même de dire cela dans le contexte où on le dit; ainsi dire à quelqu'un :

12 - Il est minuit.

c'est peut-être signifier, dans un certain contexte, qu'il doit partir immédiatement; on peut enchaîner sur un énoncé du type :

13 - Il est tard. Dépêche-toi.

comme cela peut signifier, dans un autre contexte, qu'il est trop tard pour partir, et on pourrait enchaîner cette fois sur un énoncé du type :

14 - Il est trop tard. Ce n'est pas la peine de partir maintenant.

Ce qui est signifié l'est non pas par la phrase, mais par le fait de son énonciation. Dans les deux cas, l'énoncé 12 est orienté vers le tard; or, on peut facilement imaginer un autre contexte dans lequel le même énoncé peut être orienté vers le tôt :

15 - Tu peux partir. Il est minuit. 
Il faut noter ici que l'orientation de cet énoncé vers le tôt n'est pas facilement compréhensible abstraction faite du contexte d'énonciation. À cet effet, les éléments prosodiques au niveau de l'oral, comme le ton ou l'intonation, par exemple, sont d'un grand intérêt. Ce que la négation restrictive rend plus explicite : 16 - Il n'est que minuit. Tu peux partir.

Il en va de même pour l'ordre d'apparition des deux propositions au niveau de l'énoncé (15) : il ya une certaine mise en valeur de la première proposition " tu peux partir » servant de conclusion par rapport à la deuxième proposition « il est minuit » qui sert d'argument. "Tu peux partir " implique qu'il y a toujours du temps, ce qui justifie que " il est minuit " considéré comme argument est orienté vers le tôt. Ainsi, l'énoncé « il est minuit » ne signifie pas nécessairement par son contenu représentatif, mais signifie aussi en fonction de son insertion dans telle ou telle situation d'énonciation.

Notons aussi qu'un argument définit toujours une classe de contre-arguments, comme une conclusion définit une conclusion inverse. Un discours argumentatif se place toujours par rapport à un contre-discours effectif ou virtuel.

Dans un discours argumentatif, l'enchainement des énoncés est intrinsèquement lié à leur nature et fondé non pas sur les états du monde auxquels ils renvoient, mais sur leur sens. Or, il est central dans la théorie argumentative que le sens d'un énoncé comporte une allusion à son éventuelle continuation : il lui est " essentiel » d'appeler tel ou tel type de suite, de prétendre orienter le discours dans telle ou telle direction. Comprendre le sens d'un énoncé, c'est comprendre ce vers quoi " pointe " cet énoncé, la visée intentionnelle de son interlocuteur.

Bref, la théorie de l'argumentation se détache donc de cette conception vériconditionnelle du langage qui réduit le sens des énoncés à leur valeur de vérité. Elle s'attache en revanche à ce qu'Anscombre et Ducrot présentent comme un «structuralisme du discours idéal ${ }^{9}$ ", théorie selon laquelle une entité linguistique tire toute sa réalité du discours où elle prend place non pas de

$\overline{9}$ Jean-Claude Anscombre et Oswald Ducrot, op. cit. 
celui auquel elle est empiriquement incorporée, mais de celui qu'elle exige, qu'elle revendique, car c'est cette revendication qui la constitue ${ }^{10}$.

Revenons maintenant à la thèse qui consiste à considérer comme optimiste celui qui dit que, et cela abstraction faite de tout contexte d'énonciation, le verre est à moitié plein et à " accuser " de pessimiste celui qui dit que le verre est à moitié vide.

Considérons donc les énoncés a et b, comme exemples correspondant respectivement aux deux attitudes (optimiste et pessimiste) :

a - Ce verre est à moitié plein.

b - Ce verre est à moitié vide.

Comment récuser une telle thèse? Tel est l'objet de notre intervention.

\section{Quelques arguments}

Tout d'abord, il faut se rendre à l'évidence : les deux prédicats " être à moitié plein " et " être à moitié vide " respectivement dans les exemples (a) et (b) sont le résultat de deux procès exprimés nécessairement par les verbes remplir et vider considérés comme des verbes d'action. Les verres ne "naissent " pas à moitié pleins ou à moitié vides, ils le deviennent. Vider et remplir sont des verbes d'action qui supposent un devenir, un procès (abstraction faite de son aspect achevé ou en cours).

L'idée de procès est donc indispensable pour l'interprétation de tels énoncés. En effet, les énoncés (a) et (b) donnent lieu à deux procès apparemment opposables : ce verre est vide, on le remplit ou bien ce verre est plein, on le vide. Disons donc que nous avons affaire au procès de remplissage et au procès de vidage. Ceci étant, on ne peut énoncer (a) :

a - Ce verre est à moitié plein.

que si l'on a affaire à un procès de remplissage, autrement dit que si l'on est en train de remplir le verre. Cet énoncé ne peut pas

$\overline{10}$ Oswald Ducrot, Les échelles argumentatives, Paris, Minuit, 1980. 
être produit alors que l'on est en train de vider le verre. Dans (a), le prédicat " être à moitié plein " exige ou revendique le procès de remplissage avec lequel il semble être compatible.

Il en va de même pour l'énoncé (b) :

$\mathrm{b}$ - Ce verre est à moitié vide.

Un tel énoncé est complètement naturel si l'on comprend bien que l'on est en train de vider le verre, mais il devient bizarre par rapport au procès de remplissage : en remplissant un verre, il semble difficile de décrire ce verre par un prédicat relatif au procès de vidage à savoir " être à moitié vide ".

Comme, nous l'avons déjà signalé, le prédicat « être à moitié plein » exige ou revendique un procès de remplissage, le prédicat " être à moitié vide " exige ou revendique un procès de vidage. Bref, l'énoncé (a) introduit généralement l'idée que le verre, au moment où l'énoncé est prononcé, est en train de se remplir. Si l'on remplaçait "à moitié plein " par "à moitié vide ", l'effet serait inverse, et le verre devrait être en cours de vidage, d'où l'énoncé (b).

On peut imaginer facilement un contexte qui explicite davantage l'idée du procès de remplissage ou du procès de vidage :

- Remplissez entièrement ce verre, il n'est qu’à moitié plein. ou inversement :

- Videz complètement ce verre, il n'est qu'à moitié vide.

"Remplissez entièrement ce verre " est compatible avec " il n'est qu'à moitié plein » et non avec "il n'est qu'à moitié vide »; de même "videz complètement ce verre " semble compatible avec " il n'est qu'à moitié vide " et non avec "il n'est qu’à moitié plein ». C'est la raison pour laquelle les énoncés suivants semblent complètement bizarres :

-*Remplissez entièrement ce verre, il n'est qu’à moitié vide. ou inversement :

-*Videz complètement ce verre, il n'est qu’à moitié plein.

Notons donc que la phrase (a) appartient à l'échelle argumentative orientée vers les trois quarts pleins. Or, cette échelle détermine à 
son tour une orientation homologue dans la graduation objective relative au nombre de centilitres pouvant remplir le verre.

La phrase (b), au contraire, appartient à l'échelle argumentative orientée vers les trois quarts vides. Cette échelle, elle aussi, détermine à son tour une orientation homologue dans la graduation objective relative au nombre de centilitres pouvant vider le verre.

Dans le schéma qui suit, nous attribuerons au verre une contenance précise (20 centilitres). Il s'agit d'un simple artifice de présentation : son but est de nous permettre d'utiliser des expressions différentes pour désigner des degrés de graduation physique et ceux de l'échelle argumentative :
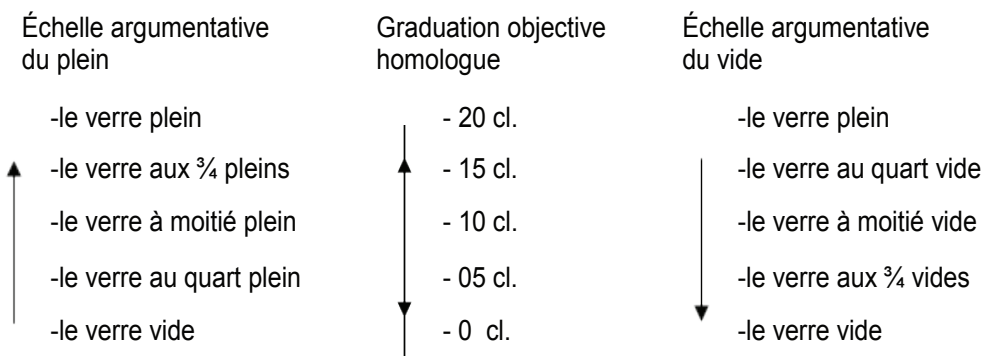

De ce qui précède, on peut donc conclure que le fait de prononcer les énoncés (a) et (b) ne revient pas à dire que l'on est respectivement " optimiste » et "pessimiste». La production de tels énoncés dépend essentiellement du procès de remplissage ou du procès de vidage que les prédicats " être à moitié plein " ou " être à moitié vide » exigent ou revendiquent. Cela dépend donc de l'orientation argumentative de chaque énoncé : ici de l'orientation vers le plein pour l'énoncé (a) et de l'orientation vers le vide pour l'énoncé (b).

Cette idée de procès est même indiquée par les énoncés (a) et (b) par ce qu'ils signifient sur le plan implicatif. Décrire ces énoncés nous incite tout d'abord, dans le cadre de l'implication logique, à parler de l'hypothèse minimaliste ${ }^{11}$. Celle-ci consiste, par exemple, à soutenir que :

11 Jean-Claude Anscombre et Oswald Ducrot, op. cit. 
Ali a 20 ans et moi 30 ans.

ou encore :

Omar a bu un litre.

signifie qu'Ali a au moins 20 ans, que moi j'ai au moins 30 ans, et qu'Omar a bu au moins un litre. Ainsi :

Le verre est à moitié plein.

ou

Le verre est à moitié vide. signifie que :

a’ - Le verre est au moins à moitié plein. ou

b’ - Le verre est au moins à moitié vide.

Plus généralement, selon cette hypothèse minimaliste, les phrases (a) et (b) excluent les phrases supérieures (c'est-à-dire plus que la moitié) comme :

* A - Le verre est aux trois quarts plein, ... plein.

* B - Le verre est aux trois quarts vide, ... vide.

Ce phénomène se prévoit facilement du point de vue implicatif, selon lequel la phrase supérieure implique par définition l'inférieure, ce qui permet d'écrire (a) $\rightarrow$ (a') et (b) $\rightarrow$ (b').

Ce n'est pas fini : on peut expliciter davantage cette idée de procès en ajoutant " encore " aux énoncés (a) et (b) :

$a^{\prime \prime}$ - Ce verre est encore à moitié plein.

b” - Ce verre est encore à moitié vide.

Concernant "encore ", on sait bien qu'il existe généralement deux acceptions temporelles principales : une valeur itérative qui marque la répétition d'un événement :

- C'est lui qui a encore téléphoné?

- Non, cette fois c'était son frère.

On pourrait imaginer également un contexte dans lequel " encore », utilisé dans (a") et (b"), exprime une valeur itérative 
(contexte dans lequel le locuteur remarque qu'on lui sert, le plus souvent, la moitié du verre) :

a” - Encore une fois, le verre à moitié plein!

b”' - Encore une fois, le verre à moitié vide!

"Encore » exprime aussi une valeur continuative qui marque la persistance d'un état ou d'une action. En effet, la description sémantique de l'énoncé (a") doit y reconnaître, entre autres choses, les deux indications suivantes :

1 - Ce verre est à moitié plein.

2 - Ce verre ne sera pas à moitié plein ultérieurement.

Ces indications montrent bien que l'on a affaire à un procès de remplissage ou de vidage. L'énoncé « a : Ce verre est à moitié plein. " ne peut être dit que si on est dans un processus de remplissage, alors que l'énoncé «a” : Ce verre est encore à moitié plein. " ne peut s'utiliser que lorsque on est dans un processus de vidage. L'adverbe " encore ", nous semble-t-il, inverse le processus, (et non l'état "d'être plein »), auquel on a affaire avant l'introduction de cet adverbe. En effet, l'énoncé « a : Ce verre est à moitié plein. ", considéré comme un argument, peut servir la conclusion : "Remplissez-le entièrement! » Mais, en introduisant " encore », on aura l'énoncé " a" : Ce verre est encore à moitié plein. " qui sert d'argument pour une conclusion inverse comme "Videz-le complètement! ", ce qui permet de conclure que " encore » joue, ici, le rôle d'un inverseur argumentatif à l'instar de l'adverbe « justement ${ }^{12}$ ». Or, "le verre est encore à moitié plein " a le même aspect optimiste que "le verre est à moitié plein ", même si ces deux énoncés, nous l'avons déjà dit, sont utilisés quand on a affaire à deux processus opposables : respectivement celui de vidage et de remplissage. Qu'est-ce qui se passe exactement? La réponse, nous semble-t-il, c'est que " encore " n'inverse que le processus (ici de remplissage); il n'inverse pas l'état "d'être plein » connotant l'optimisme. Ici, " encore "

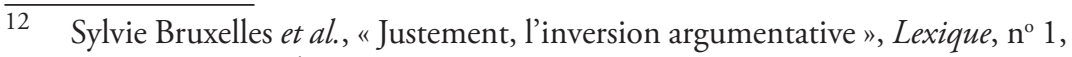
1982, p. 151-164. 
exprime une valeur continuative qui marque la persistance de cet état " d'être plein».

Il en va de même pour (b”). Les deux indications suivantes doivent être reconnues au niveau de sa description sémantique :

1 ' - Ce verre est à moitié vide.

2' - Ce verre ne sera pas à moitié vide ultérieurement.

Ces indications soulignent bien que l'on a affaire à un procès de vidage ou de remplissage : l'énoncé « b : Ce verre est à moitié vide. ", ne peut être dit que si on est dans un processus de vidage, alors que l'énoncé " b" : Ce verre est encore à moitié vide. ", ne peut s'utiliser que lorsque on est dans un processus de remplissage. L'adverbe " encore ", nous semble-t-il, inverse le processus, (et non l'état " d'être vide»), auquel on a affaire avant l'introduction de cet adverbe. En effet, l'énoncé « b : Ce verre est à moitié vide. ", considéré comme un argument, peut entraîner la conclusion : "Videz-le complètement! ». Mais, l'énoncé «b” : Ce verre est encore à moitié vide. " sert d'argument pour une conclusion inverse comme "Remplissez-le entièrement!» Or, «le verre est encore à moitié vide " a le même aspect pessimiste que " le verre est à moitié vide ", même si ces deux énoncés sont ulisés quand on a affaire à deux processus opposables : respectivement celui de remplissage et de vidage. Qu'est-ce qui se passe encore ici? Le même commentaire que nous venons de faire pour les énoncés a et a": " encore " n’inverse que le processus (ici de vidage); il n'inverse pas l'état " d'être vide " connotant le pessimisme. Ici, l'adverbe " encore " exprime une valeur continuative qui marque la persistance de cet état "d'être vide ".

En général, tout énoncé du type « $\mathrm{x}$ est encore dans l'état $\mathrm{E}$ au moment $\mathrm{t}$ » indique à la fois un posé : " $\mathrm{x}$ est dans l'état $\mathrm{E}$ en $\mathrm{t}$ " (indication déjà contenue dans l'énoncé avant l'introduction de " encore »), et un présupposé : «à un moment ultérieur tx, $\mathrm{x}$ ne sera pas dans l'état $\mathrm{E} »$

Un deuxième argument, en faveur de la nature fonda-mentalement argumentative de la langue, permet de récuser davantage cette thèse " optimiste-pessimiste " relative à l'interprétation des 
énoncés en question : (a) et (b).

En disant (a) :

a - Ce verre est à moitié plein.

On affirme du même coup, et cela d'une manière implicite, que le verre dont il s'agit est à moitié vide : il n'y a pas, sur le plan logico-sémantique, de verre à moitié plein sans qu'il soit à moitié vide. Le prédicat "être à moitié plein » entretient une relation d'antonymie lexicale avec le prédicat " être à moitié vide ». Ici, l'usage du mot moitié, à lui seul, indique l'idée d'un tout (ici le verre) subdivisé en deux moitiés - une moitié pleine et une moitié vide - et l'on ne peut pas affirmer l'une sans affirmer l'autre. Notons bien qu'elles ne sont pas affirmées au même titre par (a) : l'affirmation "à moitié plein » constitue l'objet propre de l'énoncé. C'est ce dont le locuteur entend informer le destinataire; la situation est différente pour la seconde affirmation "à moitié vide " qui est impliquée par l'énoncé (a).

Il en va de même pour (b) :

$\mathrm{b}$ - Ce verre est à moitié vide.

En disant qu'un verre est à moitié vide, nous affirmons aussi que ce même verre est à moitié plein : on ne peut pas avoir un verre à moitié vide sans qu'il soit aussi à moitié plein. Certes, comme nous l'avons déjà dit, les deux affirmations ne sont pas apportées au même titre, mais elles sont toutes les deux affirmées par l'énoncé.

C'est pourquoi, un énoncé comme (c) ou (d) semble tout à fait bizarre :

${ }^{*} \mathrm{c}$ - Ce verre est à moitié plein et à moitié vide.

*d - Ce verre est à moitié vide et à moitié plein.

La bizarrerie de l'énoncé (c) tient au fait que "à moitié vide » est redondant dans la mesure où " un verre à moitié plein » est nécessairement aussi " un verre à moitié vide ». Il en va de même pour l'énoncé $(\mathrm{d})$ : «à moitié plein » est redondant du fait qu' " un verre à moitié vide " est nécessairement aussi " un verre à moitié plein ". 
On peut donc dire que l'énoncé (a) ou l'énoncé (b) affirme simultanément le plein et le vide et ce n'est pas contradictoire mais plutôt complémentaire au sens mathématique du terme. Or, le plein, aux yeux de certains, connote l'optimisme et le vide connote le pessimisme; comment peut-on donc juger que dire (a), c'est être optimiste et dire (b), c'est être pessimiste puisque ces deux énoncés en question affirment chacun les deux valeurs : le plein et le vide en même temps?

Ce n'est pas fini : un autre argument peut être présenté pour défendre l'idée que la caractérisation du verre par "à moitié plein " ou par "à moitié vide " ne doit pas représenter "l'optimisme " ou le pessimisme ", et cela même si l'on admet que l'idée du plein connote "l'optimisme », celle du vide, «le pessimisme».

En effet, ceux qui prétendent que l'idée du plein, en général, symbolise l'optimisme et l'idée du vide, le pessimisme, le prétendent abstraction faite du contenu du verre! La contenance n'a aucune valeur en elle-même; c'est plutôt le contenu qui doit être considéré : le verre est plein de quoi? Ou encore le verre est vide de quoi? C'est de ce « quoi » que doit dépendre normalement la représentation ou bien positive ou bien négative du plein ou du vide. En effet, comment peut-on admettre de "l'optimisme " quand on parle d'un verre plein de quelque chose qui représente une valeur négative? Le plein, dans ce cas, requiert une signification " pessimiste " comme lorsque l'on dit, à titre d'exemples : plein de microbes, plein de saletés, plein de risques, etc. En revanche, le vide peut, à son tour, dans certains contextes, avoir une connotation positive, donc " optimiste " comme dans cet exemple :

-Ne craignez rien, la ville est vide (nettoyée) de brigands. ou encore :

- Heureusement que cette place est vide! J'en ai besoin.

Notons que certaines entités quantifiées ne semblent pas pouvoir accepter "à moitié " :

-*Ce verre est à moitié plein d'eau.

-*La ville est à moitié vide de brigands. 
-*Il est à moitié plein de microbes.

Dans ce type d'exemples, les adjectifs (plein et vide) n'acceptent pas, nous semble-t-il, d'être suivis d'un complément et d'être précédés en même temps de "à moitié "; de deux choses l'une : ou bien ces adjectifs sont suivis d'un complément :

- Ce verre est plein d'eau.

- La ville est vide de brigands.

- Il est plein de microbes.

ou bien, ils (plein et vide) sont précédés de "à moitié " :

- Ce verre est à moitié plein.

- La ville est à moitié vide.

- Il est à moitié plein.

Abstraction faite de ces contraintes syntaxiques, on peut facilement affirmer que ce qui compte le plus ici, ce n'est pas la contenance (à moitié plein / à moitié vide), mais c'est plutôt le contenu : plus le contenu a une valeur positive, plus il requiert une valeur " optimiste "; moins le contenu a une valeur positive, plus il requiert une valeur " pessimiste ».

La théorie argumentative rend compte de cela : en effet, dans les exemples ci-dessus, le terme " plein " acquiert une valeur positive orientant vers une conclusion positive (d'où sa connotation optimiste), mais cette orientation positive ou cet optimisme se heurte à des compléments d'adjectifs comme "brigands ", " microbes " qui ont une valeur négative, donc négativement orientés (d'où leur aspect pessimiste). Il en va de même pour le mot "vide " : orienté négativement, ce mot connote du pessimisme, mais associé à un complément comme "brigands " ou à un adverbe modificateur comme " heureusement ", le mot "vide " enclenchant une connotation négative requiert une autre orientation positive, donc optimiste, qui est due à l'association du mot "vide " aux termes "brigands » et " heureusement» comme dans les exemples :

- Ne craignez rien, la ville est vide (nettoyée) de brigands. 
- Heureusement que cette place est vide! J'en ai besoin.

Par ailleurs, le contenu lui-même peut susciter beaucoup de divergences qui émanent de la différence ou de la diversité des représentations culturelles qu'on se fait de telle ou telle chose (ce n'est l'objet de cette intervention pour que je puisse donner plus de détails).

Il faut remarquer ici que le fait d'associer les valeurs culturelles aux entités quantifiées par "à moitié ...." ne relève pas des préoccupations de Ducrot. Cette association nous permet de remettre en question la thèse psychologisante susmentionnée.

Il faudrait toutefois signaler qu'une description objective et soucieuse de la complexité des éléments engendrant la production des énoncés du langage, ne devrait pas se limiter aux seuls éléments inhérents à la langue. Une telle description pourrait également prendre en considération certains aspects extralinguistiques qui pourraient être à l'œuvre et pertinents du point de vue interprétatif. Bref, les valeurs d'optimisme ou de pessimisme, effectivement et spontanément associés aux énoncés produits dans des situations réelles de communication, ne doivent pas être exclues de l'interprétation même si ces dernières ne sont pas de nature purement linguistique. Les exemples suivants montrent bien à quel point ces connotations sont enracinées dans l'usage des locuteurs et des scripteurs :

- " mieux vaut une tête bien faite que bien pleine " (Montaigne).

- « avoir les mains pleines".

- " l'estomac plein ressemble à une conscience satisfaite " (Hugo).

- " plein aux as " (très riche).

- " rentre les mains vides».

- " propos vides".

- " la nature a horreur du vide».

- « ce vide nauséeux" (Duhamel).

- « ce vide mortel de ces heures sans projets " (Martin du Gard). 


\section{Conclusion}

D'un point de vue purement linguistique, on peut conclure, d'une part, que les énoncés (a) et (b) :

a - Ce verre est à moitié plein.

b - Ce verre est à moitié vide.

n'impliquent pas que l'on est " optimiste " ou que l'on est " pessimiste ", une fois qu'on les utilise! En revanche, leur usage dépend essentiellement du procès en cours auquel on a affaire : dire (a) exige que l'on est en train de remplir le verre au moment où on le prononce, comme dire (b) exige que l'on est en train de vider le verre au moment où on le dit. On ne peut pas affirmer (a) alors que l'on est en train de vider le verre ou inversement affirmer (b) alors que l'on est en train de remplir le verre. Dire (a), ce n'est donc pas " être optimiste ", comme dire (b) ce n'est pas « être pessimiste ».

D'autre part, les prédicats " être à moitié plein " ou " être à moitié vide " sont simultanément affirmés soit par l'énoncé (a) soit par (b); cela signifierait, selon les " psychologues ", que l'on est "optimiste et pessimiste " en même temps, ce qui semble logiquement contradictoire.

Enfin, la valeur positive ou la valeur négative représentées respectivement par l'optimisme et le pessimisme ne dépend pas nécessairement de la contenance (plein ou vide), mais plutôt de la nature du contenu. Comparons un texte plein de fautes et un pays plein d'or ou un mot vide de sens et une rue vide d'escrocs.

Par ailleurs, cela ne permet pas nullement de dénier les valeurs d'optimisme ou de pessimisme associées à certains énoncés produits dans des situations réelles de discours. D'autres paramètres extralinguistiques peuvent intervenir dans l'interprétation de ce type de connotations. Tel est ce que nous voulons et pouvons proposer! 


\section{Bibliographie}

Anscombre, Jean-Claude et Oswald Ducrot, L'argumentation dans la langue, Bruxelles, Mardaga, 1983.

Bruxelles, Sylvvie et al., " Justement, l'inversion argumentative ", Lexique, $n^{\circ}$ 1, 1982, p. 151-164.

Ducrot, Oswald, Dire et ne pas dire. Principes de sémantique linguistique, Paris, Herman, 1972.

Ducrot, Oswald, Les échelles argumentatives, Paris, Minuit, 1980.

Morris, Charles W., Foundations of the Theory of Signs, Chicago, University of Chicago Press, 1948.

Roulet, Eddy, Langue maternelle et langues secondes : vers une pédagogie intégrée, Hatier-Crédif, 1980. 